\title{
Design and development characteristics research of modular space deployable solar wing
}

\author{
Shuai Yang ${ }^{1, *}$, Qiong $\mathrm{Wu}^{2}$, and Boyan Chang ${ }^{1}$ \\ ${ }^{1}$ School of Mechanical Engineering, Tianjin Polytechnic University, Tianjin 300387, China \\ ${ }^{2}$ School of Mechanical Engineering and Automation, Beihang University, Beijing 100191, China
}

\begin{abstract}
In order to solve the problems of the existing solar wing, such as complex process and obvious structural vibration. a new space deployable solar wing mechanism composed of single DOF modularization was designed. The structure and working principle of the space deployable solar wing are described, and the driving system is briefly introduced. The single module of the solar wing is divided into two parts and the kinematics equations of the kite-shaped mechanism and origami mechanism are established respectively. Secondly, the output motion rule of the bar at the end was solved when the special driving is applied to the kite-shaped mechanism. And based on this output, changed the angle of the origami mechanism' plates and the change curve of the centre of mass and the angle between the plates is obtained. The study results show that, synthesize considering the shrinkage and the ratio of folding to span, the optimal solution is the angle of plates $\varphi=4^{\circ}$. Through the above research that provide the theoretical basis for the optimization and improvement of the mechanism.
\end{abstract}

\section{Introduction}

The solar wing (solar array) was the main energy source of the satellites. It was usually used to supply energy for satellites and spacecraft. In order to satisfy the enveloped size limit of the carrier, the launch section was generally in a closed state and pressed against the cabin of the spacecraft by mechanical devices. After the spacecraft enters orbit, the solar wing was deployed and maintained in a stable operating state [1-2].

The space deployable solar wing had been studied by several people over the past time. Chen et al took a certain type of satellite solar wing drive mechanism as the research object, analysed the vibration question during the operation, and obtained the formation principles of two disturbance frequencies [3]. Hou et al used the counterweight suspension method to carry out a horizontal deployment test on the satellite solar wing surface after the gravity was eliminated, which laid the foundation for the actual operation of the solar wing in orbit [4]. Zhang et al proposed a geometric modelling method for rotating solar sails, using ABAQUS software to simulate the dynamics of the solar sail during the second stage of

${ }^{*}$ Corresponding author: yangshuai_tj@163.com 
deployment, and verified the correctness of the proposed theory [5]. Ding et al proposed a method of using a solar array driven by a root hinge, established a multi-DOF dynamic model of the system, and verified the control effect of the drive assembly through ground tests. The results showed that the developed solar array able to successfully complete the deployment process [6].

This paper proposed a modular space deployable solar wing mechanism, and that expounds the structure principle and driving mode, set up the kinematics model of kiteshaped mechanism and origami mechanism, through a given input motion law, solving the origami institutions change curve of angle and the centroid position, provide the theoretical basis for further research and application.

\section{Structure composition and working principle of solar wing}

\subsection{Structure composition}

The space deployable solar wing was designed with modular design. It was composed of a body rack, driving system and several monomer modules superimposing. The monomer module includes kite-shaped mechanism and origami mechanism. The driving system was integrated in the body rack, and the whole driving system was completely separated from the execution part, the structure diagram is shown in Fig. 1. The kite-shaped mechanism plays the role of motion transmission and support. As the main working surface, origami mechanism can install solar plates of the same specification and size to collect solar energy, as shown in Fig. 2.

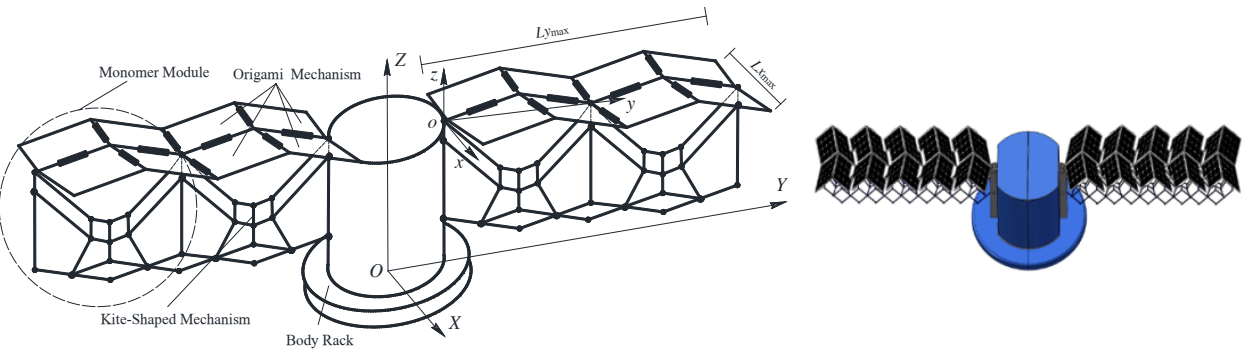

Fig. 1. Schematic diagram of space deployable solar wings.

Fig.2. The 3D model of solar wing.

For the whole solar wing mechanism, the centre point at the bottom of the body rack was taken as the origin $O$, and the axis $Z$ was perpendicular to the bottom of the body rack. The stacking direction of the monomer module at the right end was selected as the positive direction of the axis $Y$, and the axis $X$ is along the normal direction of the monomer module and conforms to the right-hand rule, so as to establish the basic coordinate system $O X Y Z$. At the same time, based on the modular composition principle of the mechanism, the modular superposition method was divided into two types. One was to increase the number of monomer modules in the axis $Y$ direction; The other was to increase the number of origami mechanism in the axis $X$ direction. In the Fig. 1, $L x_{\max }$ and $L y_{\max }$ respectively represented the maximum expansion length of the two directions under the condition of a specific number of modules. Meanwhile, the shape and size of origami plates were the key factors affecting the maximum expansion length. 


\subsection{Operating principle of the driving system}

Face up to the plane $Y O Z$ shown in Fig. 1, analyze the working principle of the driving system, can be intuitive, whole driving system along the plane XOZ of symmetry, as shown in Fig. 2, The motor provides power and controls the expansion speed. When the matching nut moves down along the rectangular lead screw, the slider rail end of the driving link slides in the sliding guide rail until the end actuator expands in the direction of axis $\pm Y$. At the same time, the rectangular screw nut can lock the mechanism at any position to ensure good expansion precision and drive. The advantages of this solar wing are flexible topological structure, and the number and size of modules can be changed according to the actual requirements. Due to the modular design idea, there are fewer types of components, high interchangeability, and high storage rate and working area.

\section{Kinematics analysis of kite-shaped mechanism}

The kite-shaped mechanism was extracted from the single module shown in Fig. 1, and the motion characteristics of the mechanism were analyzed. The included angles of each member and axis were $\theta_{1} 、 \theta_{2} 、 \theta_{3} 、 \theta_{4}$ and $\theta_{5}$, as shown in Fig. 4.
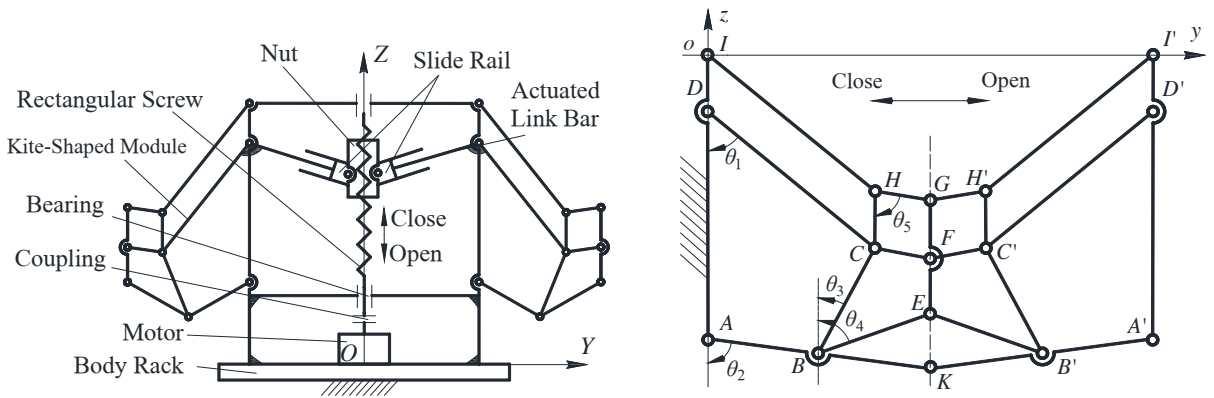

Fig.3. Schematic diagram of driving system.

Fig.4. Schematic diagram of kite-shaped mechanism.

All the bars of the kite-shaped mechanism are connected by revolute joint, in which the shortest $C F$ bar is taken as the unit length, and the other bars satisfy the following expression

$$
\left\{\begin{array}{l}
L_{C H}=L_{G H}=L_{C F} \\
L_{B C}=L_{B E}=L_{E G}=\frac{1}{k} L_{C F} \\
L_{A K}=L_{C D}=L_{H I}=\frac{1}{k^{2}} L_{C F} \quad k=0.5 \\
L_{A I}=\frac{1}{\left(k^{2}+1\right)} L_{C F}
\end{array}\right.
$$

The kinematic modeling process of kite-shaped mechanism was omitted here. For details, referred to References [7]. Selecting $\theta_{1}=4.27^{\circ}$ as the initial angle, the highest cubic input motion law, when $t=0 \mathrm{~s} \sim 10 \mathrm{~s}$, the expression is as follows

$$
\theta_{1}=4.27^{0}+ \begin{cases}\frac{15 \pi t^{2}-2 \pi t^{3}}{459} & 0 \leq t \leq 5 \\ \frac{125 \pi \times\left[1-3 \times\left(\frac{\mathrm{t}}{5}-1\right)^{2}-2 \times\left(\frac{\mathrm{t}}{5}-1\right)^{3}\right]}{459} & 5 \leq t \leq 10\end{cases}
$$


The length of the $C F$ bar is $200 \mathrm{~mm}$, according to the above rod length proportional relationship, and the driving expression of formula (2) was substituted into kinematic equations, the movement law curve of the middle $E G$ bar in the two axial directions can be obtained, as shown in Fig. 5. Due to the complete symmetry of the mechanism, the kinematic characteristics of the end $A^{\prime} I^{\prime}$ bar can be deduced. Its value in the axis $z_{1}$ direction does not change, and it moves strictly in a straight line along the positive direction of axis $y_{1}$. And use this feature as the basis of driving the paper folding mechanism, provide the four-plates paper folding mechanism with one end fixed, and the other end for linear unfolding movement.

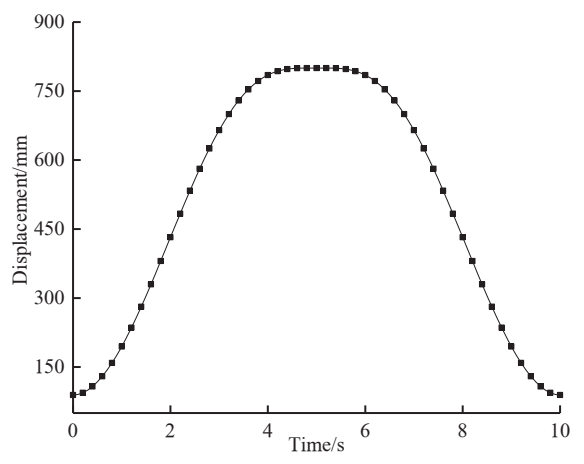

Fig. 5. Motion curve of the middle member of the kite-shaped mechanism.

\section{Kinematics analysis of origami mechanism}

The origami mechanism was further extracted from the single module, and the local coordinate system oxyz is established at the rotation point $O$, and each parameter symbol is calibrated, as shown in Fig. 6, so as to analyze the movement characteristics of the plate.The prototype model was established here, as shown in Fig. 7, which verifies the correctness and feasibility of the mechanism.
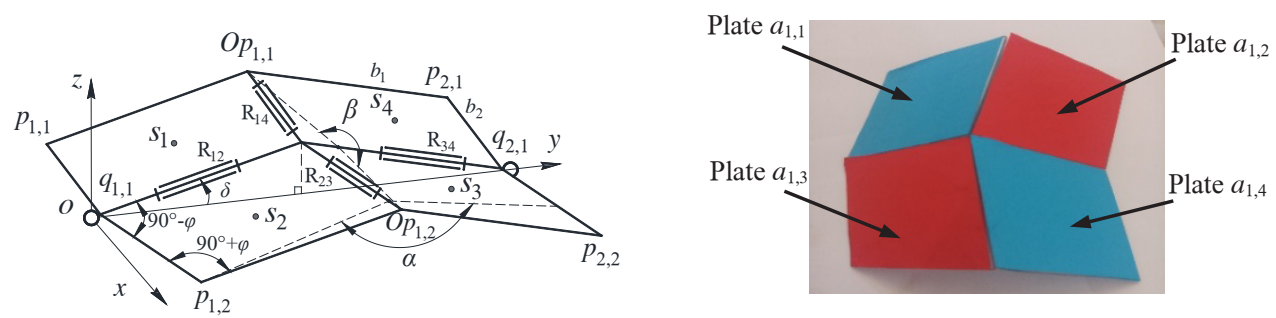

Fig. 6. Schematic diagram of origami mechanism. Fig. 7. Prototype diagram of Origami mechanism.

It should be noted that the origami mechanism is composed of four identical homogeneous parallelogram plates. The angle relationship on plane yoz was obtained as follows

$$
\delta=\arccos \left(\frac{y_{E G}}{b_{1}}\right)
$$

For the combination $O q_{1,1}$, take point $q_{1,1}$ as the coordinate origin $o$, the straight line of point $q_{1,1}$ and point $q_{2,1}$ as the axis $y$ and the axis $x$ along the normal direction of plane $N_{q-1}$ 
and comply with the right-hand rule, establish the coordinate system as shown in Fig.8. Among them, $\boldsymbol{u}$ and $\boldsymbol{v}$ were the vertical segments from point $q_{1,1}$ and point $q_{2,1}$ to the straight line $O p_{1,1} \sim O q_{1,1}$, the corresponding line lengths are $u$ and $v$, the distance between the two perpendicular feet was $d$, and the displacement of point $q_{2,1}$ relative to point $q_{2,1}$ was $s$. The dihedral angle formed by translating $\boldsymbol{u}$ to $\boldsymbol{u}^{\prime}$ on member $a_{1,1}$ is $\alpha$.

According to the dihedral angle formula

$$
d=\sqrt{s^{2}-u^{2}-v^{2}+2 u v \cos \alpha}
$$

It can be deduced that the expression $\alpha$ is

$$
\alpha=\arccos \left(\frac{d^{2}-s^{2}+u^{2}+v^{2}}{2 u v}\right)
$$

where

$$
d=2 b_{1} \sin \varphi ; \quad v=u=u^{\prime}=b_{1} \cos \varphi
$$

The origami mechanism is abstracted as a spherical 4R mechanism, as shown in Fig. 9. The constraint formula of the same length of the bar is established by using the space coordinate transformation method, and the kinematic expression of the mechanism is obtained. The mathematical modeling process is referred to References [8].

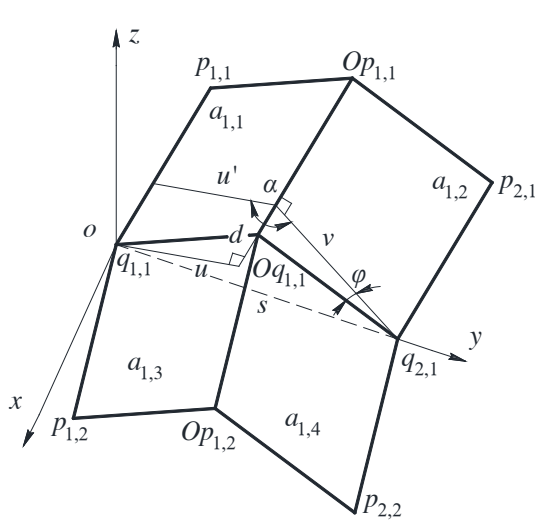

Fig. 8. Schematic diagram of assembly $O q_{1,1}$

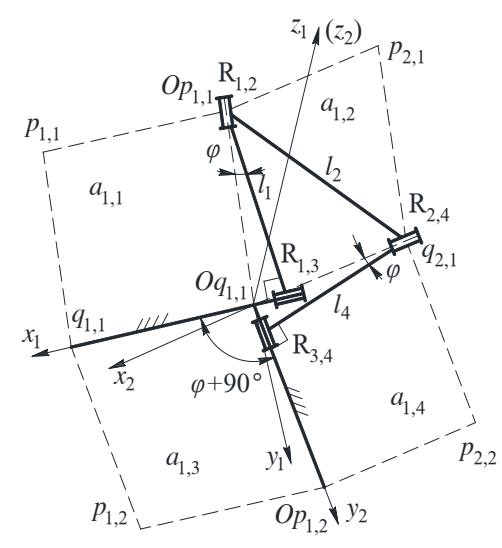

Fig. 9. Schematic diagram of the equivalent spherical 4R mechanism of assembly $O q_{1,1}$

$$
U \cos \beta+V \sin \beta+W=0
$$

where

$$
\begin{aligned}
& U=-2 l_{1} l_{4} \sin \varphi(1+\cos \alpha) ; \quad V=-2 l_{1} l_{4} \sin \alpha ; \\
& W=\frac{\left(l_{1}^{2}+l_{4}^{2}\right)}{\cos ^{2} \varphi}-l_{2}^{2}-2 l_{1} l_{4}\left[\frac{\tan \varphi}{\cos \varphi}-(1+\cos \alpha) \sin \varphi\right]
\end{aligned}
$$

According to formula (6), it can be solved

$$
\beta=2 \arctan \frac{V \pm \sqrt{U^{2}+V^{2}-W^{2}}}{U-W}
$$

When the angle $\alpha$ changes continuously in the range of $0^{\circ} \sim 180^{\circ}$, the negative sign should be selected in formula (7) according to the position and assembly relationship of the mechanism. The angle change of parallelogram plate is analyzed. As shown in Fig. 9, when 
the black, red and blue contours are selected as $\varphi=2^{\circ} 、 4^{\circ} 、 6^{\circ}$, the parameters and centroid position will change accordingly.

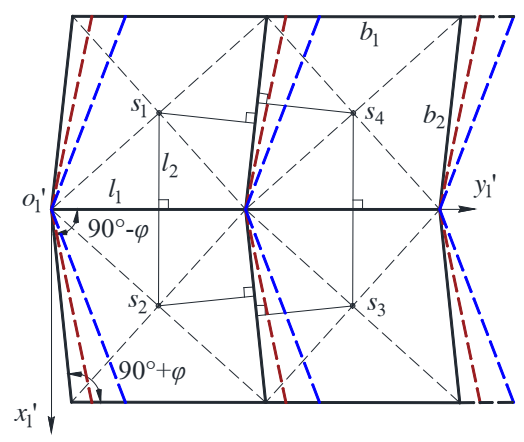

Fig. 9. Schematic diagram of full expansion of origami mechanism.

The expression for the distance $l_{1}$ and the length of the vertical segment $l_{2}$ are

$$
\left\{\begin{array}{l}
l_{1}=\frac{b_{1}(1+\tan \varphi)}{2} \\
l_{2}=\frac{b_{1}}{2}
\end{array}\right.
$$

The position coordinates of centroids $s_{1}$ and $s_{2}$ are

$$
s_{1}=s_{2}=\left\{\begin{array}{l}
x_{1}= \pm \frac{l_{2}}{\cos \varphi} \sin \left(\frac{\alpha}{2}\right) \\
y_{1}=\left(l_{1}-l_{2} \tan \varphi\right) \cos \delta+\frac{l_{2}}{\cos \varphi} \cos \left(\frac{\beta}{2}\right) \\
z_{1}=\left(l_{1}-l_{2} \tan \varphi\right) \sin \delta
\end{array}\right.
$$

The position coordinates of centroids $s_{3}$ and $s_{4}$ are

$$
s_{3}=s_{4}=\left[\begin{array}{l}
x_{1} \\
y_{E G}+y_{1} \\
z_{1}
\end{array}\right]
$$

The selection of the positive and negative signs in formula (9) and formula (10) is related to the quadrant where the position of the mass center of each plates is located. The position of the centre of mass of the origami mechanism can be calculated, which provides a theoretical basis for further analysis and optimization of dynamic characteristics.

The influence of the change of plate angle $\varphi$ shown in Fig. 10 on the operation process of the mechanism is analyzed. Among the three selected angles, when the plate angle $\varphi=6^{\circ}$ is selected, the change value of the angle between the plates of the origami mechanism is the largest, which is $\alpha=4.8^{\circ} \sim 180^{\circ}$ and $\beta=44.6^{\circ} \sim 180^{\circ}$, the maximum value can be reached in two folding directions, and the more compact the mechanism shrinks.

The motion characteristics of the center of mass of the mechanism plate shown in Fig. 11 are analyzed, and each curve is projected into a plane. Through comparison, it can be seen that when $\varphi=2^{\circ}$, the initial position of the mechanism in the $y$ direction is small, and its expansibility in the $Y$ direction is large. 


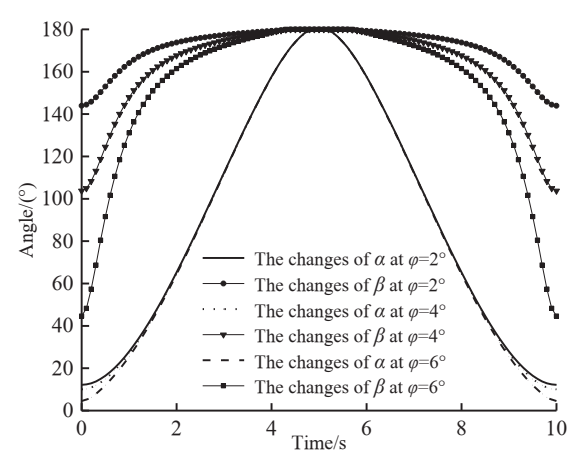

Fig. 10. Schematic diagram of driving system.

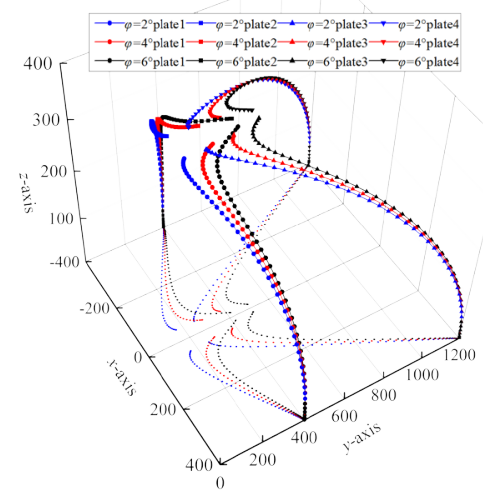

Fig. 11. Schematic diagram of driving system.

Based on the comprehensive analysis of the two motion characteristic curves and the comparison of the two extreme conditions, the intermediate value $\varphi=4^{\circ}$ should be selected as the optimal parameter solution, and the configuration optimization is made for the further analysis of the mechanism.

\section{Conclusion}

In this paper, a new type of modular developable solar wing mechanism was be propose, and introduces the composition and working principle. The kinematics models of kiteshaped mechanism and origami mechanism in single module were established respectively. When the initial motion law was given that the motion law of the end bar was obtained. And on this basis, the kinematic characteristics of origami mechanism with the change of the plate's angle were analyzed. The results show that when choice the plate angle $\varphi=4^{\circ}$, the more compact the mechanism shrinks, and the development degree in axis $Y$ direction was the largest.

\section{References}

[1]. Z. Q. Zhou, Y. M. Wu, J. Wang, et al. Development and trend of circular solar array[J]. Spacecraft Engineering, 24(06): 116-122, (2015).

[2]. M. Hu, W. J. Li, W. H. Chen, et al. Motion simulation and function test on repeated fold-unfold mechanism of fan-shaped solar array[J]. Chin J Spac Sc, 36(01): 92-98, (2016).

[3]. J. P. Chen, W. Cheng, X. Li. Modeling and simulation of the disturbance torque generated by a solar array drive assembly[J]. Journal of Vibration and Shock, 36(20): 188-194+217, (2017).

[4]. P. Hou, Z. H. Li, T. Song, et al. A novel method for horizontal deployable experiment of satellite solar array[J]. J Harbin Inst Technol, 48(10): 176-182, (2016).

[5]. X. X. Zhang, C. Y. Zhou. Dynamic analysis of spinning solar sails at deployment process[J]. CJA, 30(05): 1719-1728, (2017).

[6]. X. L. Ding, X. Li, K. Xu, et al. Study on the behavior of solar array deployment with root hinge drive assembly[J]. CJA, 25(02): 276-284: (2012).

[7]. S. Yang, B. Y. Chang, X. N. Li, et al. Dynamic analysis on the kite straight-line deployable mechanism[J]. Journal of Machine Design, 37(03): 39-46, (2020).

[8]. B. Y. Chang, S. Yang, G. G. Jin, et al. Motion Analysis of Spatial Deployable 
mechanism driven in sraight line[J]. JME, 56(05): 192-201, (2020). 